Renaissance einer alten Therapieoption

Die Behandlung von Problemwunden mit Fliegenmaden

A. Schmelz, M. Kramer, A. Beck, L. Kinzl, P. Keppler, Th. Einsiedel
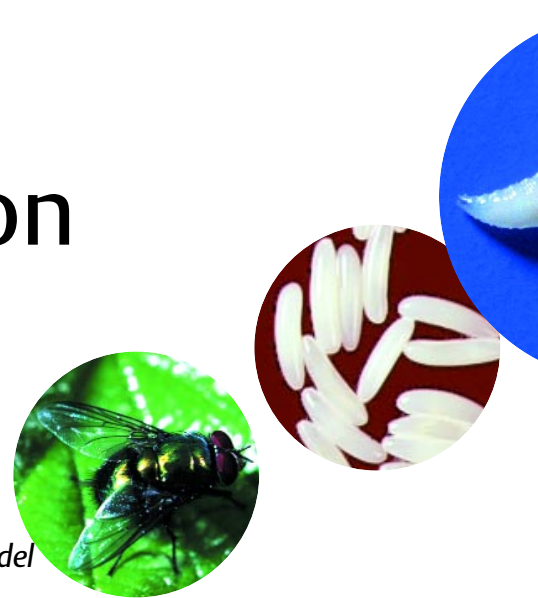

Abteilung für Unfall-, Hand- und Wiederherstellungschirurgie, Universitätsklinikum Ulm

(Ärztlicher Direktor: Prof. Dr. L. Kinzl)

klinikarzt 2004; 33 (8+9): 246-249

Auch mit Hightechmethoden der modernen Wundbehandlung sind chronische Wunden - ein Ulcus cruris, der diabetische Fuß oder Dekubitusulzera - häufig nur schwer zu therapieren. Hier hat in den vergangenen Jahren mit der Biochirurgie eine bereits „historische“ Therapieoption eine Renaissance erfahren. Auf den ersten Blick gewöhnungsbedürftig können damit gute Erfolge erzielt werden, da die heute steril gezüchteten Maden der Lucilia-sericata-Fliegen nicht nur ein sensibles Debridement des nekrotischen Gewebes gewährleisten, sondern zudem eine antimikrobielle Komponente besitzen und die Matrixproliferation stimulieren. Sicherlich kann die biochirurgische Therapie konventionelle Wundbehandlungsstrategien nicht ersetzen. Berücksichtigt man jedoch die morphologischen und pathophysiologischen Ursachen chronischer Wunden, beachtet man die Indikation und bettet man diese Option in chirurgisch basierte (interdisziplinäre) Konzepte ein, kann man eine gute Behandlungsqualität - bei geringen Kosten - erzielen.
$\mathrm{M}$ ehr als drei Millionen Menschen leiden in der Bundesrepublik Deutschland an chronischen Wunden, deren Ursache das Ulcus cruris, der diabetische Fuß oder der Dekubitus sind. Diese Art der Erkrankungen findet noch zu wenig Beachtung, wenngleich sie einen nicht unerheblichen Kostenfaktor in der Heilbehandlung darstellen: Gegenwärtig belaufen sich die Kosten auf etwa fünf Milliarden Euro pro Jahr.

\section{Häufig problematisch}

Mit 60-80\% ist das häufigste Leiden der nicht spontan heilenden Wunden das Ulcus cruris, in Deutschland sind ungefähr 800000 Menschen betroffen. Besonders zu beachten ist die hohe Rezidivrate mit über $90 \%$, was die besondere Schwie- rigkeit solcher Wundverhältnisse dokumentiert. Die Behandlungskosten liegen bei einem Patienten mit einem chronischen Ulkus bei etwa 1500 Euro pro Jahr, die durch Arbeitsunfähigkeit und Krankenhausaufenthalte entstehenden Verluste summieren sich auf bis zu eine Milliarde Euro jährlich.

Eine weitere problematische chronische Wunde ist - vor allem bei jüngeren Patienten - der diabetische Fuß. Auch in diesen Fällen ist es schwer, die Defekte effizient zu behandeln, die Folge sind Fußamputationen bei über 20000 Patienten pro Jahr. Und die Folgekosten bei jungen Patienten gehen in die Milliarden.

Probleme bereitet auch die zunehmende Überalterung unserer Bevölkerung und damit der immer größere Anteil an älteren Kranken oder pflegebedürftigen Patienten: Bis zu $40 \%$ der Menschen in stationärer und häuslicher Pflege leiden an einem Dekubitalulkus. Diese Veränderungen gehören mit zu den Herausforderungen in der Versorgung älterer Menschen. Schätzungsweise sind in dieser Altergruppe 1,5 Millionen Menschen an einem solchen Wundleiden erkrankt.

Die Therapiemöglichkeiten für solche „Problemwunden“ sind vielfältig, immer populärer wurde in letzter Zeit jedoch die Behandlung mit Fliegenmaden. Eine entscheidende Grundvoraussetzung für diese Option ist, dass sich die Maden ausschließlich von totem Gewebe ernähren. Daher verwendet man die Maden von Lucilia sericata, die als Außenverdauer bezeichnet werden.

Die stetig wachsende Popularität dieses Behandlungsprinzips hat das Interesse an Erkrankungen wie dem diabetischen Fuß, dem Ulcus cruris oder dem Dekubitus steigen lassen. Viele Kliniken haben inzwischen so genannte Wundsprechstunden eingerichtet, viele Selbsthilfegruppen beschäftigen sich mit diesem Thema. All dies hat dazu beigetragen, dass die Behandlung chronischer Wunden von einem in der Vergangenheit eher niedrigen Stellenwert mitten in den medizinischen und gesellschaftlichen Fokus gerückt ist. 
- Therapie mit Fliegenmaden

Debridement, antimikrobielle Aktivität und Granulationsförderung sind nach neuesten Erkenntnissen die wichtigsten Wirkmechanismen der Therapie mit Fliegenmaden. Die Komplexität ist einmalig und reiht sich hervorragend in das biologische System der Wundbehandlung ein. Die positiven Erfahrungen der Behandlung chronischer Wunden mit dem Wundsekret der Fliegenlarve von Lucilia sericata führte in Deutschland zu einer geradezu explosionsartigen Ausweitung der Therapieindikation, oftmals leider verbunden mit einer falschen Indikationsstellung.

\section{Debridement der Wunde}

Um Nekrosen zu beseitigen, werden in der Regel chirurgische Hilfsmittel herangezogen. Dabei besteht jedoch die Gefahr, dass die Grenze zwischen nekrotischem und gesundem Material unzureichend beurteilt wird und es somit zu einer Defektvergrößerung kommt. Die Fliegenmaden dagegen sind ein wesentlich sensibleres chirurgisches Instrument: Sie verteilen ihr Sekret auf der Wundoberfläche, welches eine Reihe proteolytischer Enzyme enthält, und die Nekrosen werden verflüssigt. Dieser Mechanismus ermöglicht den Maden, abgestorbenes Gewebe als Nährbouillon aufzunehmen.

Innerhalb von drei Tagen verzehnfachen die Maden ihre Körperlänge, indem Sie diese verflüssigten Nekrosen aufnehmen. Dies wiederum bedeutet, dass das nekrotische Gewebe effizient abgebaut wird ohne dass das gesunde Gewebe wesentlich beeinträchtigt wird - ein grenznekrotisches Debridement (Abb. 1).

\section{Antimikrobielle Therapie}

In der Regel sind chronische Wunden bakteriell kontaminiert. Treten die Erreger von der Nekrose auf vitales Gewebe über, kommt es unter Umständen zur Ausbildung von Erysipelen, Phlegmonen und Lymphangitiden, was wiederum eine Sepsis mit lebensbedrohlicher Komplikation auslösen kann. Ziel einer jeden Behandlung muss daher

\section{Abb. 1 Grenznektorisches Debridement}
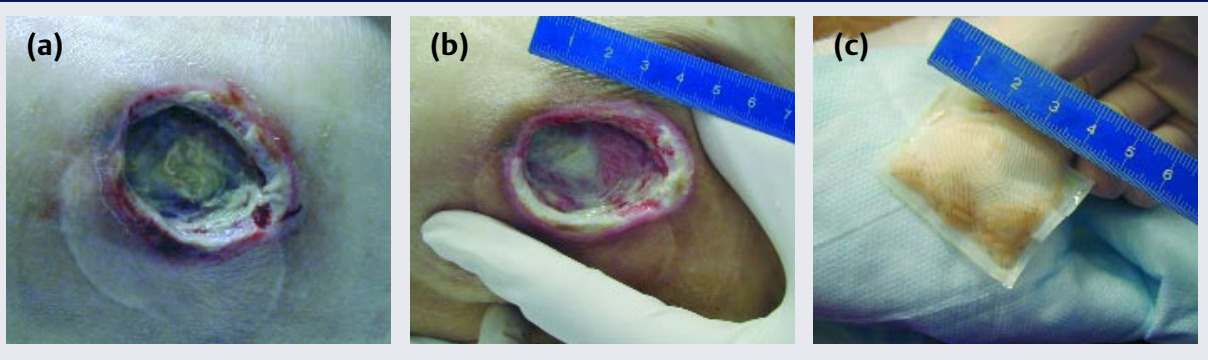

Dekubitus am Trochanter vor (a) und nach (b) dem fünftägigen Debridement mit Lucilia-sericata-Maden. Nach den fünf Tagen haben die Maden ihre Körpergröße etwa verzehnfacht auch die Vermeidung einer Wundinfektion über den oberflächlichen Bereich hinaus sein. Betrachtet man die zunehmende Resistenzentwicklung vieler Bakterienstämme auf effiziente Antibiotika, ist es umso wichtiger, lokale Wundbehandlungsmaßnahmen in ihrer Wirksamkeit zu steigern.

Schon in den 30er Jahren des letzten Jahrhunderts beschrieben Robinson et al eine antimikrobielle Aktivität von Fliegenmaden. Diese Wirkung ist auf im Madensekret vorkommende Faktoren zurückzuführen, wie 30 Jahre später Pavillard et al dokumentierten. Spezielle Untersuchungen untermauern diese antibiotische Aktivität des Luciliasericata-Sekrets, welches auch bei methicillinresistenten Staphylokokken wirksam zu sein scheint.

Noch sind die Mechanismen, die zu solchen Effekten führen, nicht geklärt. Möglich scheint die Expression alkalisierender Substanzen, welche eine bakterielle Besiedlung erschweren oder unmöglich machen. Ursächlich sind unter anderem auch die im Darmsekret von Maden nachgewiesenen Substanzen wie Phenyl- acetat und Phenacetaldehyd. Wahrscheinlich existieren jedoch weitere aktive Substanzen, welche noch nicht bekannt sind, die für diesen Mechanismus verantwortlich gemacht werden müssen.

Neben dem Madensekret führt auch die intestinale Aktivität dieser Tiere zu einer Keimreduktion. Mumcuoglu hat erst vor kurzem beschrieben, dass Maden farbstoffmarkierte E.-coli-Bakterien aufgenommen und metabolisiert haben. Zudem hinterlassen Fliegenmaden bei der Besiedlung von Bakterienrasen bakterienfreie Spuren (Abb. 2). Die Aufarbeitung der ausgesetzten Maden auf dem Bakterienrasen belegte, dass im Darm der Tiere diese Bakterien zumeist in lysierter Form identifiziert werden konnten.

\section{Stimulation der Matrix- proliferation}

Zunächst wurde diskutiert, dass die Proliferation von Zellen auf die mechanische Reizung des Wundgrundes durch die behaarte Oberfläche der Fliegenlarven zurückzuführen sei. Kürzlich wurde jedoch gezeigt, dass das Wachstum von hu-

\section{Abb. 2 Antibiotische Aktivität des Lucilia-sericata-Sekrets}

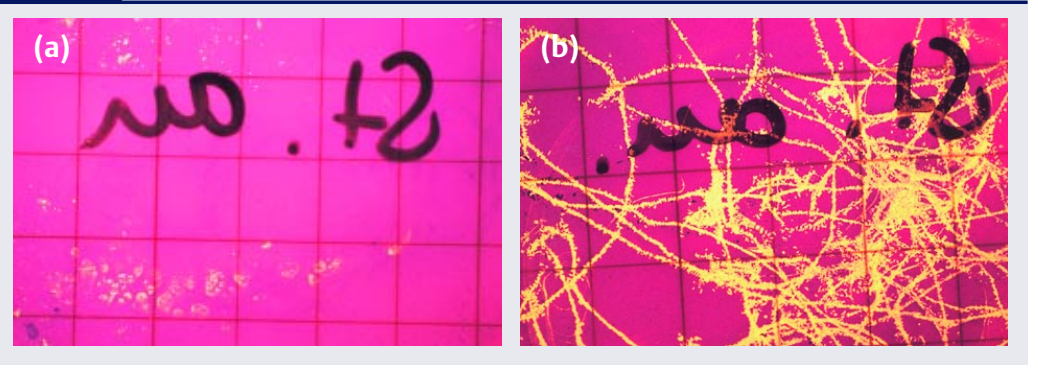

Vor der Exposition mit den Maden ist der Staphylococcus-Biofilm noch intakt (a), nach Madenexposition (zehn Tiere, zehn Minuten) sind deutliche bakterienfreie Spuren zu sehen (b) 


\section{Abb. 3 Stimulation der Matrixproliferation}

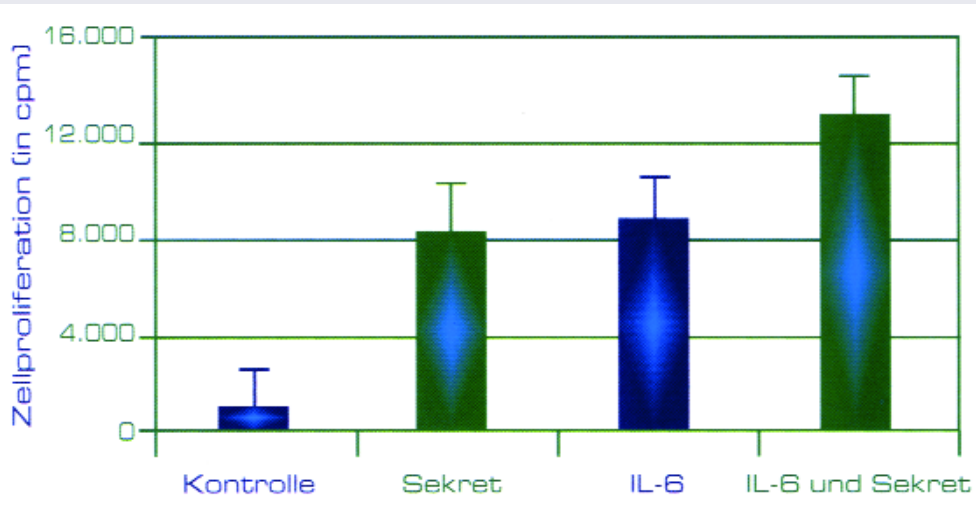

manen Fibroblasten durch die Expression des Sekrets der Lucilia sericata stimuliert wird. Gleichzeitig konnte gezeigt werden, dass das Sekret der Fliegenlarven die fibroblastenstimulierende Wirkung von Interleukin-6 (IL-6) sowie IGF (,insulin-like growth factor") verstärkte (Abb. 3).

\section{Eigene Erfahrungen \\ mit der Biochirurgie}

Unserer Erfahrung nach lassen sich durch die Behandlung von Problemwunden mit sterilen Fliegenmaden Behandlungsergebnisse erzielen, die auch mit Hightechmethoden in der modernen Wundbehandlung nur schwer zu erreichen sind. Zwischen 1998 und 2000 wurden in der Unfallchirurgischen Abteilung der Universitätsklinik Ulm $57 \mathrm{~Pa}$ tienten mit Fliegenlarven behandelt. Dabei handelte es sich um therapie-

\section{Abb. 4 Diabetisches Fußulkus}

(a)

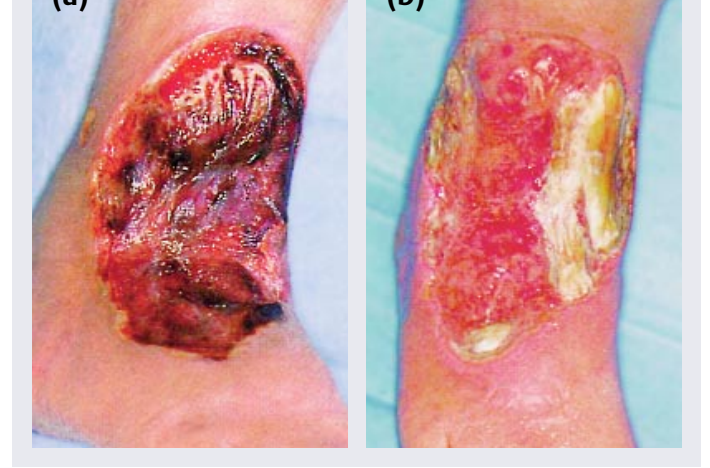

Ein diabetisches Ulkus vor dem Einsatz von 200 Fliegenmaden (a). Nach vier Tagen (b) war das Ulkus nahe komplett debridiert resistente Wunden, das Patientenkollektiv wies einen Altersdurchschnitt bei den Männern um 59 und bei den Frauen um 48 Jahre auf. Zur Infektbeherrschung und Vermeidung der Extremitätenamputation wurde die Madentherapie indiziert.

\section{Methodik}

Zunächst wurden die Wunden mit Ringer-Lösung gereinigt und soweit möglich chirurgisch debridiert. Danach wurden die Fliegenmaden auf die Wundoberfläche aufgebracht und durch saugfähige Kompressen abgedeckt. Ein solcher luftdurchlässiger saugfähiger Verband ist notwendig, um die Maden in der Wunde nicht zu ersticken.

In der Regel dauert die Behandlung pro Madentherapie drei bis vier Tage, danach werden die Maden erneuert. Im Mittel sind pro Patienten drei Anwendungen nötig, in unserem Patientenkollektiv belief sich die Anzahl der Behandlungen zwischen einer und 17 Madenapplikationen.

\section{Ergebnisse}

Das beste Behandlungsergebnis wurde bei frischen bzw. posttraumatischen Wunden erzielt. In diesen Fällen waren auch die wenigsten biochirurgischen Behandlungszyklen notwendig - in der Regel reichten hier ein bis zwei Applikationen aus. Therapieversager wurden nicht beobachtet. Oftmals konnte das einliegende Osteosynthesematerial bei festem Sitz belassen werden, die Madentherapie führte zu einem raschen Abheilen der Infektwunden, und bei entsprechender Knochen- konsolidierung konnte eine vorzeitige Metallentfernung durchgeführt werden.

Chronische Wunden mit chirurgischer Vorbehandlung sprachen ebenfalls sehr gut auf die Madenbehandlung an. Gerade diabetische Defektwunden mit verminderter Wundheilungskapazität am Fuß zeigten unter der Madentherapie ein gutes Abheilungsvermögen, zuvor abgelaufene chirurgische Behandlungen konnten diese Erfolge nicht aufweisen. Bereits nach zwei bis sechs Behandlungszyklen waren die Wunden vollständig abgeheilt.

Bei der Behandlung von Dekubitalulzerationen lag die Indikationsstellung der Madentherapie weniger in der Infektsanierung allein als vielmehr in der Unterstützung derselben. Hier kam es nach durchschnittlich zwölf Behandlungen zu einer deutlichen Wundsäuberung und zu einer Stimulation der Granulationsbildung, was die Grundlage für plastisch-chirurgische rekonstruktive Maßnahmen bildete.

Die schlechtesten Ergebnisse bzw. keine Heilung konnten bei Patienten mit chronischen Wunden infolge einer arteriellen Verschlusskrankheit im Stadium IV beobachtet werden - unabhängig davon, ob ein begleitender Diabetes vorlag oder nicht.

\section{Kein Ersatz, aber Therapie- ergänzung}

Die Behandlung von chronischen oder akut infizierten Wunden mit Fliegenmaden ersetzt sicher nicht die Therapie von Chirurgen, Internisten, Dermatologen und Mikrobiologen. Diese Option ist aber eine sinnvolle Ergänzung der Therapie in den Fällen, in denen die anerkannten Standardtherapien mit den herkömmlichen Antibiotika versagt haben und als Ultima ratio nur noch weit greifende verstümmelnde Maßnahmen bis hin zur Amputation möglich scheinen.

Wichtig ist beim Einsatz dieses Therapeutikums, dass die kausale Therapie der zugrunde liegenden Erkrankung berücksichtigt wird. Es ist jederzeit möglich, die Biochirurgie in eine interdisziplinäre Therapie einzubetten. Durch die Möglichkeit 
der ambulant stationären Behandlung kann ein patientenorientiertes Behandlungskonzept im Hinblick auf Behandlungsqualität, Patientenkomfort, aber auch auf die Kosten entwickelt werden. Diese Therapie kann die Dauer des Krankenhausaufenthalts reduzieren, durch die ambulante häusliche Betreuung können Krankenhauseinweisungen vermieden sowie der Einsatz von Antibiotika reduziert werden.

\section{Indikationen}

Die Kombination aus chirurgischem Debridement und dem Einsatz von Fliegenmaden scheint eine effiziente Behandlungsalternative zur bisherigen Behandlung chronischer Wunden zu sein. Eine gute Indikation stellt die chronische Wundinfektion, insbesondere die diabetische Wundheilungsstörung dar (Abb. 4). Schlechte Ergebnisse finden sich bei der arteriellen Verschlusskrankheit (AVK) im Stadium IV, eine verzögerte Effizienz ist bei kontaminierten Sehnen, Bändern und Faszien zu beobachten. Hier kann die Effizienz durch begleitende chirurgische Maßnahmen erheblich gesteigert werden.

Durch den Einsatz der Fliegenmaden werden unnötige Gewebedefekte vermieden, da die so genannten Biochirurgen mit ihrem hochaktiven Wundsekret sehr exakt zwischen lebendem und totem Gewebe unterscheiden können und damit gewebeschonender debridieren als der Chirurg mit seinem Skalpell. Auch die antimikrobielle Wirkung zeigt die hohe Kompetenz beim Einsatz der Fliegenmaden, die neben der Infektbeseitigung auch eine hervorragende Stimulation der Wundheilung zeigt. Es kommt nämlich zu einer raschen Auffüllung der Defektwunde durch Granulationsgewebe, gleichzeitig setzt eine strapazierfähige Epithelialisierung der Wundoberfläche in Verbindung mit einer Wundverkleinerung durch Wundkontraktion ein.

\section{Bislang keine Neben- wirkungen}

Bei sachgemäßem Einsatz dieser Therapieform sind bislang keine Nebenwirkungen bekannt. So ist die
Übertragung von Keimen durch die Maden nahezu ausgeschlossen, da sie steril gezüchtet werden. Da man heute Fliegenmaden nur in „ausbruchssicheren" Wundauflagen (Biobag BioMonde) verwendet, ist zudem der „Verlust“ von Maden aus der Wunde mit Kreuzinfektionen oder die ungewollte Verbreitung dieser Maden auf stationärer Ebene nahezu unmöglich. Aufgrund dieser Schutzmaßnahmen ist die Anwendung von Fliegenmaden in der Behandlung von Risikowunden kein Hygienerisiko mehr, die Maden können in diesen Verbandsstoffen problemlos entsorgt werden.

\section{Gazit}

Berücksichtigt man die morphologischen und pathophysiologischen Ursachen chronischer Wunden ist die Madentherapie eine geeignete Ergänzung der bislang üblichen chirurgisch basierten Therapie von Wundbehandlungen. Bei richtiger Indikationsstellung und regelhafter therapeutischer Beziehung zwischen der Wunde und Made kann eine gute Behandlungsqualität garantiert werden.

\section{Renaissance of an Ancient Thera- peutic Option - Treatment of Problem Wounds with Maggots} Even with modern high-tech methods applied to wound management, chronic wounds - leg ulcer, diabetic foot or decubitus ulcers - are often difficult to treat. Over the past few years, biosurgery has resuscitated a therapeutic option that had long been relegated to the history books. At first glance taking some getting used to, the application of sterile maggots of the greenbottle fly Lucilia sericata achieves good results: not only do the maggots debride necrotic tissue exclusively, but they also have an antimicrobial effect, and stimulate matrix proliferation.

This biosurgical treatment can certainly not replace conventional treatment strategies. However, if we consider the morphological and pathophysiological causes of chronic wounds, and also give consideration to the indication, the option, embedded within surgery-based (interdisciplinary) concepts, can provide good-quality treatment at low cost.
Die Historie der Wundbehandlung mit Fliegenmaden

Chon vor Jahrtausenden wurde die Wundbe$\checkmark$ handlung mit lebenden Fliegenmaden praktiziert. Auch in der Zeit des Mittelalters wurden Maden in den Wunden von Kriegsverletzten beobachtet, eine gezielte Anwendung wurde jedoch nie beschrieben. Im Jahr 1829 beschrieb der französische Chirurg Larrey, dass Maden einer bestimmten Fliege nur totes Gewebe entfernen und eine positive Wirkung auf die Wundheilung haben. Etwa 100 Jahre später, nämlich 1928, implementierte der amerikanische Chirurg William S. Baer die Fliegenmaden in der Zivilchirurgie und behandelte jugendliche Patienten, die an Osteomyelitis erkrankt waren. Er beobachtete unter anderem ein schnelles Debridement, Keim- und Geruchsreduktion, eine Alkalisierung des Wundmilieus und eine ideale Heilungsrate. Gleichzeitig wies er auf die Gefahr von durch Maden induzierten Infektionen (z.B. Clostridien) hin. Die Notwendigkeit, sterile Fliegenmaden zu produzieren und zu verwenden, schien unumgänglich.

Die Einführung der flächendeckenden Antibiotikatherapie und deren phänomenale Ergebnisse bedeuteten jedoch zunächst das schlagartige Aus für die bereits weltweit angewandte Madenbehandlung. Erst Anfang der 90er Jahre etablierten die amerikanischen Ärzte Ronald Sherman und Edward Pechter eine keimfreie Fliegenaufzucht zur Gewinnung keimfreier Maden, 1995 wurde aufgrund der Therapieerfolge von Sherman und Pechter in England eine weitere Produktionsstätte unte Steve Thomas eingerichtet. Seither wurde in diversen wissenschaftlichen Publikationen auf die Madentherapie hingewiesen, sie hat zum heutigen Tage einen festen Platz bei der Behandlung von Problemwunden.

\section{Key Words}

leg ulcer - diabetic foot decubitus ulcer - biosurgical treatment - antimicrobial effect matrix proliferation

\section{Literatur bei den Verfassern}

\author{
Anschrift des Verfassers \\ Dr. Andreas Schmelz \\ Abteilung für Unfall-, Hand- \\ und Wiederherstellungschirurgie \\ Universitätsklinikum Ulm \\ Steinhövelstr. 9 \\ 89075 Ulm
}

